\title{
A COUNTABLY DISTRIBUTIVE COMPLETE BOOLEAN ALGEBRA NOT UNCOUNTABLY REPRESENTABLE
}

\author{
JOHN GREGORY ${ }^{1}$
}

\begin{abstract}
It is proved from the Continuum Hypothesis that there exists an $\omega$-distributive complete Boolean algebra which is not $\omega_{1}$-representable.
\end{abstract}

Karp [1, Corollary to main theorem] shows that there is an $\omega$ distributive non- $\left|2^{\omega}\right|$-representable $\left|2^{\omega}\right|$-complete Boolean algebra. Karp later pointed out that, for every cardinal $K \geqq\left|2^{\omega}\right|$, there is an $\omega$-distributive non- $\left|2^{\omega}\right|$-representable $K$-complete Boolean algebra. The following strengthens the completeness requirement.

TheOREM 1. The Continuum Hypothesis implies the existence of an $\omega$-distributive non- $\omega_{1}$-representable complete Boolean algebra.

In [3, Definition 1.2], the property $P_{K}$ was defined for cardinals $K$. We will prove the following theorem.

THEOREM 2. If $\left|2^{\omega}\right|<\left|2^{\omega_{1}}\right|$, then there exists an $\omega$-distributive complete Boolean algebra that does not have the property $P_{\omega_{1}}$.

Theorem 1 is a consequence of Theorem 2, since the Continuum Hypothesis implies both $\left|2^{\omega}\right|<\left|2^{\omega_{1}}\right|$ and (by [3, Theorem 4.2]) the equivalence of $\omega_{1}$-representability with property $P_{\omega_{1}}$.

Assuming the negation of Souslin's Hypothesis, Smith's [3, Theorem 3.4] implies our theorems. However, R. Jensen has shown that Souslin's Hypothesis is consistent with the Continuum Hypothesis; so Smith's result does not imply ours.

1. Preliminaries. Let $\omega$ be the first infinite cardinal; $\omega_{1}$ is the first uncountable cardinal; $x^{y}$ is the set of all functions from $y$ into $x ;|x|$ is the cardinal of $x$ (cardinals are initial ordinals); $\operatorname{Dm}(x)$ is the domain of function $x ; x \uparrow y$ is the restriction of a function $x$ to the domain $\operatorname{Dm}(x) \cap y$. We assume the axiom of choice.

Received by the editors November 15, 1972 and, in revised form, February 7, 1973. AMS (MOS) subject classifications (1970). Primary 06A40; Secondary 04A30.

Key words and phrases. Complete Boolean algebra, $\omega$-distributive Boolean algebra, $\omega_{1}$-representable Boolean algebra, Continuum Hypothesis.

${ }^{1}$ This research was partially supported by a National Science Foundation Grant of Carol Karp, NSF GP 34033.

(C) American Mathematical Society 1974 
The Boolean algebraic definitions are essentially those of [2]. A Boolean algebra $C$ is ordered by: $a \leqq b$ iff the infimum $a \wedge b=a . C$ is $K$-complete if the infimum (or meet) $\wedge S$ exists for each set $S$ of no more than $K$ many elements of $C$. Then the supremum (or join) $\vee S$ exists for all such $S$. $C$ is complete if the infimum of every set of elements exists. A $K$-complete Boolean algebra $C$ is $K$-distributive iff

$$
\bigwedge_{\alpha<K} \bigvee_{\beta<K} A(\alpha, \beta) \leqq \bigvee_{H \in K^{K}} \bigwedge_{\alpha<K} A(\alpha, H(\alpha))
$$

for all functions $A$ into $C$. A Boolean algebra is $K$-representable iff it is isomorphic to the quotient of a $K$-field of sets by a $K$-ideal. Smith defined the following distributive-like property $P_{K}$ which implies $K$ representability.

Definition. A Boolean algebra $C$ has the property $P_{K}$ iff: if $A$ is a function from $K \times K$ into $C$ and if $\bigwedge_{\alpha<K} \bigvee_{\beta<K} A(\alpha, \beta)$ exists and is not zero, then there is a function $H$ from $K$ into $K$ such that, for all $\gamma<K$, either $\bigwedge_{\alpha<\gamma} A(\alpha, H(\alpha))$ does not exist or else is not zero.

A subset $S$ of $C$ is dense iff $S$ does not contain the zero of $C$ and, for all nonzero elements $b$ of $C$, there exists $x \in S$ such that $x \leqq b$.

The following is well known and is related to "forcing".

A set $S$ ordered by $\leqq$ satisfies

for all $x \leqq y$, there exists $z \leqq x$ such that, for all $w$, not both $w \leqq y$ and $w \leqq z$

if and only if there exists an (algebraically unique) complete Boolean algebra $C$ such that $S$ is a dense subset of $C$ and the ordering of $C$ extends that of $S$. (See [2, Example 12(B)] and [2, §35].)

If $S$ is dense in $C$ and $x, y, z$ range over $S$, then: $x \leqq \wedge X$ iff, for all $b \in X, x \leqq b ; x \leqq$ complement of $b$ iff, for all $z$, not both $z \leqq x$ and $z \leqq b$; $x \leqq \bigvee X$ iff, for all $y \leqq x$, there is $z \leqq y$ and $b \in X$ such that $z \leqq b$.

2. Proof of Theorem 2. Assume $\Omega=\left|2^{\omega}\right|<\left|2^{\omega_{1}}\right|$. Suppose there did not exist an $\omega$-distributive complete Boolean algebra not having property $P_{\omega_{1}}$. It will suffice to reach a contradiction. Let $X$ be the set of all nonempty countable sequences $f$ into $2^{\omega}$ (i.e., of all functions $f$ whose nonempty domain is a countable ordinal and whose range is a subset of $2^{\omega}$ ).

Define $F$ to be the set of all $F$ such that: $F$ is a function from a subset of $X$ into $X$; if $F(f)$ defined, then $\operatorname{Dm}(F(f))=\operatorname{Dm}(f)+1$; if $F(f)$ defined, then so is $F(f\lceil\alpha)=F(f) \uparrow(\alpha+1)$ whenever $0<\alpha \leqq \operatorname{Dm}(f)$.

There exists a $\Omega$-sequence $E$ that enumerates the set of all ordered pairs $(f, t)$ such that $f \in X$ and countable $t \subseteq X$, each such pair occurring $\Omega$ times in the sequence $E$. 
By induction on $\alpha \leqq \Omega, F_{\alpha}$ will now be constructed such that $F_{\alpha} \in \mathscr{F}$, $\left|F_{\alpha}\right| \leqq|\omega \cup \alpha|$, and $F_{\beta}$ extends $F_{\alpha}$ whenever $\alpha<\beta \leqq \Omega$.

For limit ordinal $\lambda \leqq \Omega$, let $F_{\lambda}$ be the union of all $F_{\alpha}, \alpha<\lambda$. (In particular, $F_{0}$ is empty.)

Given $F=F_{\alpha}, \alpha<\Omega, G=F_{\alpha+1}$ is defined by cases as follows. In each case below, note that $G \in \mathscr{F}, G$ extends $F$, and $G$ has only countably more elements than $F$. (Cases 1, 2, 3 are for Lemmas 1(1), 1(2), 2 respectively.)

Case 1. $E(\alpha)$ is some $(f, 0)$ such that $\operatorname{Dm}(f)=2$. Since $\left(2^{\omega}\right)^{1}$ has cardinality greater than $F_{\alpha}$, there is $h \in\left(2^{\omega}\right)^{1}$ such that $F(h)$ is not defined. Extend $F$ to $G$ by: $G(h)=f ; G(k)=F(k)$ whenever defined.

Case 2. $E(\alpha)$ is some $(f,\{g\})$ such that $f$ has a domain $\gamma+1, g$ has a domain $\delta<\gamma$, and $F(g)=f \uparrow(\delta+1)$. Then there is $h \in\left(2^{\omega}\right)^{\gamma}$ such that $h$ extends $g$ and $F(h \uparrow(\delta+1))$ is not defined. Then $F(h \uparrow \beta)$ is defined iff $0<\beta \leqq \delta$; for such $\beta, h \uparrow \beta$ is $g \uparrow \beta$. Extend $F$ to $G$ by: $G(h \uparrow \beta)=f \uparrow(\beta+1)$ whenever $\delta<\beta \leqq \gamma ; G(k)=F(k)$ whenever defined.

Case 3. $E(\alpha)$ is some $(f, t)$ such that: $f$ has a domain $\gamma+1$; for every $k \in t, F(k) \subset f$ is defined; there are $\Omega$ many $h \in\left(2^{\omega}\right)^{\gamma}$ such that $h$ is a union of elements of $t$. Then there exists $h \in\left(2^{\omega}\right)^{y}$ such that $h$ is a union of elements of $t$ but $F(h)$ is not defined. Extend $F$ to $G$ by: $G(h)=f ; G(k)=F(k)$ whenever defined.

Case 4. The first three cases do not hold. Let $G$ be $F$.

This finishes the construction of $F_{\alpha}, \alpha \leqq \Omega$. From now on, let $F$ be $F_{\Omega}$. Given $B \in\left(2^{\omega}\right)^{\omega_{1}}$, define $T$ to be $\{f \mid F(f) \subset B\}$.

$T$ will turn out to be a dense subset of a complete $\omega$-distributive Boolean algebra. But first some facts about $T$ will be proved.

LEMMA 1. (1) $T$ is nonempty. (2) If $g$ is in both $T$ and $\left(2^{\omega}\right)^{\delta}$ and if $\delta<\gamma<\omega_{1}$, then $g$ has at least two distinct extensions in $T \cap\left(2^{\omega}\right)^{\gamma}$. (3) If $f \in T$ and nonzero $\beta \leqq \operatorname{Dm}(f)$, then $f \uparrow \beta \in T$.

Proof. (1) For some $\alpha, E(\alpha)$ is $(B \nmid 2,0)$. By Case 1 of the construction of $F_{\alpha+1}$, there is $h$ such that $F_{\alpha+1}(h)=B \nmid 2$. Then $F(h)=B \nmid 2$ and this $h$ is in $T$.

(2) Then for some $\beta<\Omega, F_{\beta}(g)=B\lceil(\delta+1)$ is defined. For $\Omega$ many $\alpha>\beta, E(\alpha)$ is $(B \nmid(\gamma+1),\{g\})$. For each such $\alpha$, by Case 2 of the construction of $F_{\alpha+1}$, there is assigned $h \in\left(2^{\omega}\right)^{\gamma}$ such that $h$ extends $g$, $F_{\alpha+1}(h)=B\left\lceil(\gamma+1)\right.$, and $F_{\alpha}(h)$ is not defined. Then $h$ is in $T \cap\left(2^{\omega}\right)^{\gamma}$ and $h$ extends $g$. Since $F_{\alpha}(h)$ is not defined, $h$ was not assigned to a smaller $\alpha$; thus, distinct such $\alpha$ 's give distinct $h$ 's.

(3) $F(f) \subset B$. Thus $F(f)$ is defined. Since $F \in \mathscr{F}, F(f\lceil\beta) \subseteq F(f) \subset B$. Thus, $f\lceil\beta \in T$. 
LEMMA 2. If countable $t \subseteq T$ and there are $\Omega$ many $h \in\left(2^{\omega}\right)^{\gamma}$ such that $h$ is a union of elements of $t$, then there exists $h \in T \cap\left(2^{\omega}\right)^{y}$ such that $h$ is a union of elements of $t$.

Proof. For each $k \in t, F(k) \subset B$ and some $F_{\beta}(k), \beta<\Omega$, is defined. Since $\Omega$ is not cofinal with $\omega$, there exists $\beta<\Omega$ such that, for all $k \in t$, $F_{\beta}(k)$ is defined. For some $\alpha>\beta, E(\alpha)$ is $(B \uparrow(\gamma+1), t)$. Then Case 3 of the definition of $F_{\alpha+1}$ gives $h \in\left(2^{\omega}\right)^{\gamma}$ such that $h$ is a union of elements of $t$ and $F_{\alpha+1}(h)=B\lceil(\gamma+1)$. Then $F(h) \subset B$ and $h \in T$.

Order $T$ by: $f \leqq g$ iff $f$ extends $g$. Then $T$ satisfies (1) of $\S 1$. So there is a (algebraically unique) complete Boolean algebra $C$ such that $T$ is a dense subset of $C$ and the ordering of $C$ extends that of $T$.

LEMMA 3. $C$ is an $\omega$-distributive Boolean algebra.

Proof. Suppose that $A$ is a function on $\omega \times K$ into $C$. By the definition of $\omega$-distributivity and by the fact that $T$ is dense in $C$, it suffices to show for every $f \in T$ that: if $f \leqq \bigwedge_{m} \bigvee_{\alpha} A(m, \alpha)$, then there is $h \in T$ such that $h \leqq f$ and, for all $m<\omega, h \leqq A(m, \alpha)$ for some $H(m)=\alpha<K$.

Define $P(r) \in T$ for $r \in 2^{m}$ by the following induction on $m<\omega$. Let $P(0)=f$. Suppose $P(r) \leqq f$ defined for all $r \in 2^{m}$. Let $\beta(m)$ be the least upper bound of $\left\{\operatorname{Dm}(P(r)) \mid r \in 2^{m}\right\}$. Consider any $r \in 2^{m}$. Let $u$ and $v$ be the two extensions in $2^{m+1}$ of $r$. Since $P(r) \leqq f \leqq \bigvee_{\alpha} A(m, \alpha)$, there exists $g \in T$ such that $g \leqq P(r)$ and $g \leqq A(m, \alpha)$ for some $\alpha$. By Lemma $1(2)$, there exist distinct extensions $P(u)$ and $P(v)$ of $g$ such that they both have the same domain greater than $\beta(m)$.

Put $t=\left\{P(r) \mid r \in 2^{m}\right.$ for some $\left.m<\omega\right\}$. Let $\gamma=\bigcup_{m} \beta(m)$. By Lemma 2, there exists $h \in T \cap\left(2^{\omega}\right)^{\gamma}$ such that $h$ is a union of some elements of $t$. Then $h \leqq P(0)=f$. Consider any $m<\omega$. There is $r \in 2^{m+1}$ such that $P(r) \subset h$. There is $\alpha<K$ such that $h \leqq P(r) \leqq A(m, \alpha)$.

LEMMA 4. Thas an uncountable chain.

Proof. Define $A$ on $\omega_{1} \times 2$ into $C$ by

$$
A(\omega \beta+m, i)=\bigvee\{f \in T \mid f(\beta)(m)=i\}, \quad \beta<\omega_{1}, m<\omega, i<2 .
$$

By Lemma 1(2), for every $g \in T$ there is $f \leqq g$ such that $\beta \in \operatorname{Dm}(f)$ and hence $f \leqq A(\omega \beta+m, f(\beta)(m))$. Thus since $T$ is dense, it follows that each $\mathrm{V}_{i} A(\omega \beta+m, i)$ is the identity of $C$. Since $C$ is a complete $\omega$-distributive Boolean algebra, it must have property $\boldsymbol{P}_{\omega_{1}}$ (by our earlier supposition, which is used here only). By $P_{\omega_{1}}$, there exists a function $H$ on $\omega_{1}$ into 2 such that, for all $\gamma<\omega_{1}$, the infimum $\bigwedge_{\beta<\gamma} A(\beta, H(\beta))$ is nonzero. Let $J=\{f \in X \mid f(\beta)(m)=H(\omega \beta+m)$ for all $\beta<\operatorname{Dm}(f)$ and $m<\omega\}$. Then $J$ is an uncountable chain of functions. It now suffices to prove $J \subseteq T$. Consider any $f \in J$. Let $\alpha=\operatorname{Dm}(f)$ and $\gamma=\omega \alpha$. Since $T$ is dense, there exists $g \in T$, 
$g \leqq \bigwedge_{\beta<\gamma} A(\beta, H(\beta))$. Then $g(\beta)(m)=H(\omega \beta+m)$ for all $\beta<\alpha, m<\omega$; so $g\lceil\alpha$ is $f$. By Lemma $1(3), f \in T$.

For each $B \in\left(2^{\omega}\right)^{\omega_{1}}$, we now choose $T(n, B), J(n, B)$, by induction on $n<\omega$, such that $T(n, B) \subseteq X, J(n, B) \in\left(2^{\omega}\right)^{\omega_{1}}$, and $J(n, B) \mid \alpha \in T(n, B)$ for all nonzero $\alpha<\omega_{1}$.

$T(0, B)=X . J(0, B)=B$.

Suppose $T(n, B)$ and $J(n, B)$ are defined. Define $T(n+1, B)=\{f \mid F(f) \subset$ $J(n, B)\}$ (i.e., define $T(n+1, B)$ from $J(n, B)$ as $T$ was earlier defined from $B)$. By Lemma $4, T(n+1, B)$ has an uncountable branch. The union of such a branch is some element $J(n+1, B)$ of $\left(2^{\omega}\right)^{\omega_{1}}$.

LEMMA 5. If $B$ and $B^{\prime}$ are such that, for all $n<\omega, J(n, B)\lceil 1$ equals $J\left(n, B^{\prime}\right) \uparrow 1$, then $B$ equals $B^{\prime}$.

Proof. Let $B$ and $B^{\prime}$ be such functions. It suffices to prove, by induction on nonzero $\beta \leqq \omega_{1}$, that $R(\beta): J(n, B)\left\{B=J\left(n, B^{\prime}\right) \mid \beta\right.$ for all $n<\omega$. For $\beta=1, R(\beta)$ is given. For nonzero limit $\beta, R(\beta)$ follows easily from $R(\alpha)$ for all $\alpha<\beta$. Suppose $R(\beta)$ holds for nonzero $\beta$, to show $R(\beta+1)$. Consider any $n<\omega$. Let $f$ be $J(n+1, B) \uparrow \beta$. By $R(\beta), f$ equals $J\left(n+1, B^{\prime}\right) \uparrow \beta$. Then $F(f) \subset J(n, B)$ since $f \in T(n+1, B) ; F(f) \subset J\left(n, B^{\prime}\right)$ since $f \in$ $T\left(n+1, B^{\prime}\right)$. Since $F(f)$ has domain $\beta+1$, it follows that $J(n, B) \uparrow(\beta+1)=$ $F(f)=J\left(n, B^{\prime}\right) \uparrow(\beta+1)$.

By Lemma 5 , distinct $B \in\left(2^{\omega}\right)^{\omega_{1}}$ are assigned distinct sequences $\left\langle J(n, B)|1| n\langle\omega\rangle\right.$. Thus, $\left|\left(2^{\omega}\right)^{\omega_{1}}\right| \leqq\left|\left(\left(2^{\omega}\right)^{1}\right)^{\omega}\right|$. This contradicts the assumption that $\left|2^{\omega_{1}}\right|>\left|2^{\omega}\right|$. Theorem 2 is proved.

It follows from the proof of Theorem 2 that: if $\left|2^{\omega}\right|<\left|2^{\omega_{1}}\right|$, then, for some $B$, the corresponding $T$ defined above is a dense subset of an $\omega-$ distributive complete Boolean algebra not having property $P_{\omega_{1}}$.

\section{REFERENCES}

1. Carol R. Karp, Nonaxiomatizability results for infinitary systems, J. Symbolic Logic 32 (1967), 367-384. MR 36 \#2484.

2. Roman Sikorski, Boolean algebras, Ergebnisse der Math. und ihrer Grenzgebiete, Neue Folge, Band 25, Academic Press, Springer-Verlag, Berlin and New York, 1964. MR 31 \#2178.

3. E. C. Smith, A distributivity condition for Boolean algebras, Ann. of Math. (2) 64 (1956), 551-561. MR 19, 115.

4517 Jonwall Court, Columbia, South Carolina 29206

Current address: Department of Mathematics, State University of New York at Buffalo, Amherst, New York 14226 